\title{
Composición florística, estructura y condición silvicultural de las especies arbóreas en tres usos del suelo de la finca Agroecológica Tonantzín, Diriamba, Carazo, 2017
}

\section{Floristic composition, structure and silvicultural condition of tree species in three land uses of the Tonantzín Agroecological Estate, Diriamba, Carazo, 2017}

\author{
Maybel Dimaxel Reyes Aguirre ${ }^{1}$, Edwin Alonzo Serrano², Rosa María Reyes Pérez ${ }^{3}$ \\ ${ }^{1}$ Ingeniero Forestal / Graduado en la Universidad Nacional Agraria / ORCID: https://orcid.org/0000-0001-7168-1622 \\ 2 MSc. en Manejo y Conservación de los Recursos Naturales / ORCID: https://orcid.org/0000-0002-9686-4772 / edwin.alonzo@ci.una.edu.ni \\ 3 Lic. en Biología / ORCID: https://orcid.org/0000-0003-3342-6131/mreyes@ci.una.edu.ni \\ Universidad Nacional Agraria
}

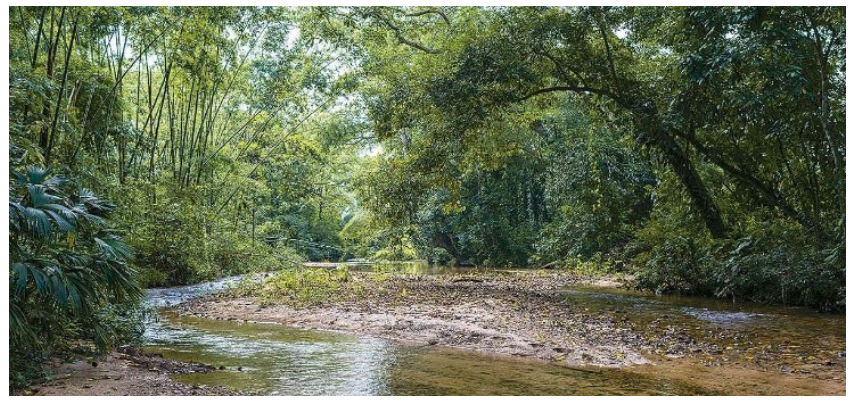

\begin{abstract}
RESUMEN
La finca agroecológica Tonantzín ubicada en el municipio de Carazo, contribuye a la conservación de la biodiversidad, el objetivo del estudio es caracterizar la composición florística estructura y condición silvicultural de especies arbóreas presentes en tres usos del suelo de la finca; bosque secundario, bosque de galería y sistema silvopastoril. La vegetación estudiada fue dividida en tres categorías de vegetación; fustales (>10 cm DN), latizal (1.5 m de altura a 9.9 $\mathrm{cm}$ de DN) y brinzal ( $0.30 \mathrm{~m}$ hasta $1.5 \mathrm{~m}$ de altura). El estudio fue llevado a cabo a través de la metodología de doble muestreo cuyo diseño tiene la forma de "L", en un área muestreada de 1.92 ha, utilizando parcelas rectangulares de $600 \mathrm{~m}^{2}$, en las cuales se midieron fustales; en parcelas cuadradas de $100 \mathrm{~m}^{2}$, latizales y brinzales en parcela cuadrada de $25 \mathrm{~m}^{2}$. Según resultados, se identificaron un total de 55 especies, agrupadas en 29 familias y 51 géneros en categoría fustal. 35 especies, 21 familias y 33 géneros en categoría latizal y 23 especies, 14 familias y 21 géneros en categoría brinzal. En fustales se registraron un total de 261.45 árboles por hectárea en bosque secundario, 347 árboles por hectárea en bosque de galería y 156.25 árboles por hectárea en sistema silvopastoril. La distribución por clase diamétrica de los árboles tuvo forma de " $\mathrm{j}$ invertida" en donde la regeneración es la que repondrá a los árboles maduros. Por otro lado, el $26.2 \%$ de los árboles presentan fuste recto sin daños ni enfermos. Así mismo el 66.5 \% se encuentran libre de lianas. Las
\end{abstract}

\begin{abstract}
The Tonantzín agroecological farm located in the municipality of Carazo, contributes to the conservation of biodiversity, the objective of characterizing the floristic composition and silvicultural condition of the tree species present in three uses of the soil of the farm; secondary forest, gallery forest and silvopastoral system. The studied vegetation was divided into three categories of vegetation: ferns (> $10 \mathrm{~cm} \mathrm{DN})$, latizal $(1.5 \mathrm{~m}$ high to $9.9 \mathrm{~cm} \mathrm{DN})$ and brinzal ( $0.30 \mathrm{~m}$ high to $1.5 \mathrm{~m}$ high). The study was carried out through the double sampling methodology of with dsing an "L" shaped design, in a sampled area of $1.92 \mathrm{ha}$. using rectangular and square plots (600 $\mathrm{m}^{2}$ ), were measured in plots, square was measured the latizal 100 $\mathrm{m}^{2}$ category and the saplings were measured in $25 \mathrm{~m}$ square plots. According to the results, a total of 55 species were grouped in 29 families and 51 genera in the category of fustales, 21 families, 33 genera and 35 species in brinzales and 14 families, 21 genera and 23 species in the brinzal category. In fustales, a total of 261.45 trees per hectare were registered in secondary forest, 347 trees per hectare in gallery forest and 156.25 trees per hectare in the silvopastoral system. The distribution by diametric class and height of the tube tree forms " $j$ inverted" where regeneration is the one that will replace the mature trees. On the other hand, $26.2 \%$ of the trees have a straight shaft without damage or illness. Likewise, $66.5 \%$ are free of lianas. The tree species with the greatest ecological importance
\end{abstract}

Recibido: 18 de febrero del 2020 Aceptado: 20 de octubre del 2020

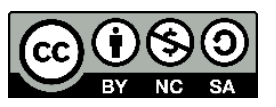

Los artículos de la revista La Calera de la Universidad Nacional Agraria, Nicaragua, se comparten bajo términos de la licencia Creative Commons: Reconocimiento, No Comercial, Compartir Igual. Las autorizaciones adicionales a las aquí delimitadas se pueden obtener en el correo edgardo.jimenez@ci.una.edu.ni

C Copyright 2020. Universidad Nacional Agraria (UNA). 
especies arbóreas con mayor importancia ecológica (IVI) presentes en los usos del suelo son: En bosque secundario Guazuma ulmifolia, en bosque de galería Ceiba pentandra y en sistema silvopastoril Enterolobium cyclocarpum.

Palabras clave: caracterizar, categorías de vegetación, vegetación arbórea.
(IVI) present in the soil uses are in secondary forest Guazuma ulmifolia, gallery forest Ceiba pentandra and silvopastoral system Enterolobium cyclocarpum.

Keywords: Characterization, vegetation categories, tree vegetation.

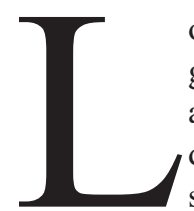

os bosques secos tropicales se caracterizan generalmente porque la mayoría de las especies arbóreas pierden su follaje en la primera mitad del período seco y muchas veces permanecen sin él durante varios meses. Su estrato superior alcanza hasta los 20 metros de altura y casi siempre existe un estrato arbustivo Lamprecht (1990).

La mayor parte de los estudios de bosques secundarios ha sido llevada a cabo en los bosques tropicales húmedos (Lamprecht, 1990). Por ello el conocimiento y evaluación de los bosques del trópico seco es un factor necesario y fundamental, para mejorar las pautas para el manejo del recurso forestal bien sea en aspectos de producción, conservación o regulación (Alvis, 2009).

En América Central quedan escasos vestigios del bosque tropical seco. Las áreas son deforestadas para ser aprovechadas como tierras agrícolas y ganadería extensiva, una vez improductivas son abandonadas y si las condiciones lo permiten, dan paso al bosque secundario, que actualmente tiene un gran interés como fuente de madera y de servicios ambientales, según el Ministerio de Recursos Naturales y del Ambiente (MARENA, 2001).

Nicaragua tiene 156966.48 ha de bosque seco, el cual se encuentra mayormente en forma dispersa en la región pacífica y central del país. Se localiza en áreas escarpadas o en áreas pedregosas y con escaso potencial agropecuario según el Instituto Nacional Forestal (INAFOR, 2009).

La finca Agroecológica Tonantzín, en Diriamba, departamento de Carazo posee pequeños fragmentos de bosques, recursos hídricos, rasgos escénicos, biológicos y ecológicos relevantes, los cuales están siendo fuertemente resguardados y conservados con la finalidad de brindar bienes y servicios ambientales, sin embargo, no existe en la propiedad un estudio de carácter ecológico y forestal, por lo cual, se ha hecho necesario realizar una caracterización general de la vegetación arbórea, los cuales, servirán a sus propietarios como base para desarrollar mecanismos y estrategias que permitan orientar de manera eficaz el manejo y conservación de la vegetación arbórea en cada uso del suelo.

\section{MATERIALES Y MÉTODOS}

El área de estudio se encuentra ubicada en la "Finca Tonantzín" en la comunidad La Trinidad, km 59 1/2 carretera
Diriamba-La Boquita, a los $11^{\circ} 43^{\prime} 57^{\prime}$ 'norte y a los $86^{\circ} 19^{\prime} 54^{\prime \prime}$ oeste, se encuentra a los $18.5 \mathrm{~km}$ del centro de Diriamba Carazo, con un área de 87.97 ha. El área de estudio limita, al norte con la comunidad la Trinidad, al sur con el río Hamayito y la finca Wascatán, al este con la finca Fátima y al oeste con la carretera hacia el río Hamayito.

Diseño metodológico. Etapa I. Planificación del inventario. Se realizó una visita al señor Humberto Solórzano, propietario de la finca Agroecológica Tonantzín, con el objetivo de abordar conjuntamente la importancia y alcance del estudio y del interés de contar con su apoyo y participación en la logística y actividades planificadas. Posteriormente, se realizó un recorrido en los sitios donde están ubicados los tres usos del suelo en la finca, para visualizar de forma general el estado en que se encuentra el área de estudio a fin de tener un escenario más claro de la situación del bosque, para definir objetivos y la metodología a emplear en el desarrollo del estudio.

Se tomó como base el mapa de la finca con la distribución de los usos del suelo. Los puntos muestreados se seleccionaron tomando como referencia puntos definidos en un estudio realizado anteriormente en la finca (Aguilar y Ramos, 2016). Existen un total de doce puntos georreferenciados y distribuidos en los tres usos del suelo, cinco en el bosque secundario, cinco en sistema silvopastoril y dos en bosque de galería.

Los cuatro puntos donde se realizó el muestreo fueron seleccionados al azar de los doce previamente definidos, eliminando la posibilidad se selección subjetiva o sesgada. Los usos del suelo y números de puntos muestreados fueron: en bosque secundario se muestrearon dos puntos, en bosque de galería un punto y en el sistema silvopastoril un punto. Para realizar el inventario forestal se utilizó la metodología de doble muestreo (Scott, 1991) con diseño de muestreo en forma de "L". Esta consistió en establecer dos líneas de inventario con dirección norte franco y este franco. En cada línea de inventario se establecieron cuatro parcelas con una distancia de $30 \mathrm{~m}$ entre cada parcela.

Clasificación de los usos del suelo y tipos de bosque de la finca agroecológica Tonantzín. Para la definición de cada tipo de bosque, uso del suelo y las categorías de uso se utilizaron los términos y definiciones de la evaluación de recursos forestales 
mundiales propuestos por la Organización de las Naciones Unidas para la Alimentación y la Agricultura (FAO, 2004), el reglamento de la ley 462 sobre conservación, fomento y desarrollo sostenible del sector forestal. Según lo anterior en la finca se encontraron 3 tipos de usos de suelos: Bosque secundario, bosque de galería y sistema silvopastoril, los cuales fueron tomados para realizar el estudio. Se clasificaron de la siguiente manera (Cuadro 1). en direcciones opuestas norte y sur franco. En cada dirección de la línea de inventario se establecieron cuatro parcelas con distancia de $30 \mathrm{~m}$ entre parcelas, dejando un margen de $10 \mathrm{~m}$ de distancia de la ribera del río. Se siguió la dirección del río considerando que en esa área tiene una dirección casi rectilínea.

Se establecieron 32 parcelas de muestreo

Cuadro 1. Clasificación del uso de la tierra y tipos de bosque de la finca agroecológica Tonantzín

\begin{tabular}{|c|c|c|c|}
\hline Tipos de bosque & $\begin{array}{l}\text { Uso del } \\
\text { suelo }\end{array}$ & $\begin{array}{c}\text { Categorías de } \\
\text { uso }\end{array}$ & Definición \\
\hline $\begin{array}{l}\text { Bosque Natural } \\
\text { Latifoliado (FAO, } \\
\text { 2004) }\end{array}$ & $\begin{array}{c}\text { Bosque } \\
\text { Secundario }\end{array}$ & Conservación & $\begin{array}{l}\text { Bosque producido por sucesión ecológica desarrollado sobre } \\
\text { tierras cuya vegetación original fue destruida por actividades } \\
\text { humanas y/o fenómenos naturales, con bajo número de } \\
\text { especies de diámetros comerciales (Reglamento de la ley } \\
462,2003 \text { ). }\end{array}$ \\
\hline $\begin{array}{l}\text { Bosque Natural } \\
\text { Latifoliado (FAO, } \\
\text { 2004) }\end{array}$ & $\begin{array}{l}\text { Bosque de } \\
\text { Galería }\end{array}$ & Conservación & $\begin{array}{l}\text { Es aquella vegetación que sobrevive por la humedad del } \\
\text { suelo y que crece por lo general frondosamente en las } \\
\text { orillas de un río (Reglamento de la ley } 462,2003 \text { ). }\end{array}$ \\
\hline $\begin{array}{c}\text { Otras Tierras (FAO, } \\
\text { 2004) }\end{array}$ & $\begin{array}{c}\text { Sistema } \\
\text { silvopastoril }\end{array}$ & Conservación & $\begin{array}{l}\text { Es una opción de producción pecuaria que involucra la presencia } \\
\text { de las leñosas perennes (árboles o arbustos), e interactúa con los } \\
\text { componentes tradicionales (forrajeras herbáceas y animales), } \\
\text { todos ellos bajo un sistema de manejo integral (Pezo e Ibrahim, } \\
\text { 1999). }\end{array}$ \\
\hline
\end{tabular}

Etapa II: Ejecución del inventario forestal. En cada punto del bosque secundario y del sistema silvopastoril se estableció el diseño de doble muestreo (Scott, 1991). Este consistió en establecer un diseño en "L" con dirección norte franco y este franco. En cada eje del diseño se delimitaron cuatro (4) parcelas con distancia de $30 \mathrm{~m}$ entre parcelas (Figura 1).

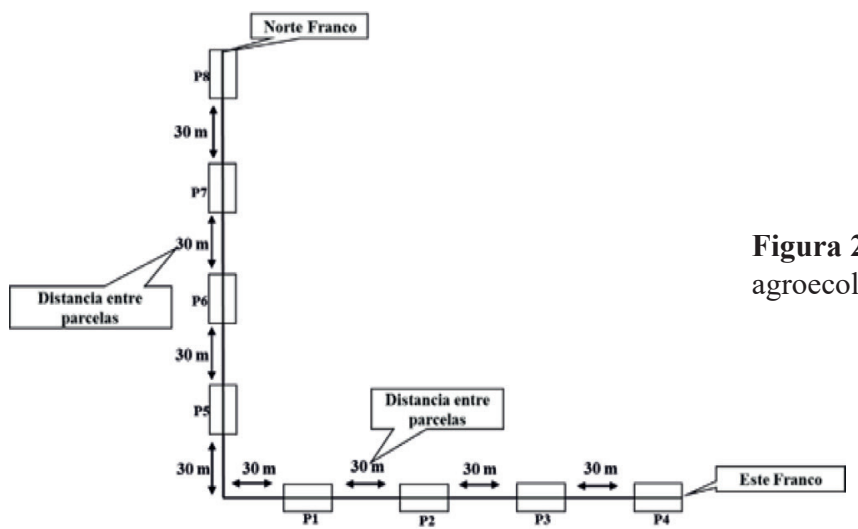

Figura 1. Diseño de muestreo utilizado en los usos del suelo (bosque secundario y sistema silvopastoril) de la finca agroecológica Tonantzín, Diriamba-Carazo, 2016.

Para el caso del bosque de galería se hizo una modificación a la metodología debido a la condición de dicho bosque (Figura 2). Consistió en establecer una línea de inventario a lo largo del rio con una distancia total de $480 \mathrm{~m}$. La línea de inventario partió de un punto centro de referencia groecológica Tonantzín, Diriamba-Carazo, 2016. distribuidas de la siguiente forma: 16 en el bosque secundario, ocho en el sistema silvopastoril y ocho en el bosque de galería. Cada parcela tuvo forma rectangular con dimensiones de $20 \mathrm{~m}$ x 30 $\mathrm{m}$. En esta se midieron todos los individuos $\geq 10 \mathrm{~cm}$ de diámetro normal considerados como fustales (Sáenz y Finegan, 2000). Cada una de estas parcelas se dividió en cuatro cuadrantes para establecer de forma aleatoria una subparcela de $10 \mathrm{~m} \times 10 \mathrm{~m}$ para contabilizar los una subparcela de $5 \mathrm{~m}$ x $5 \mathrm{~m}$ para contabilizar los brinzales.

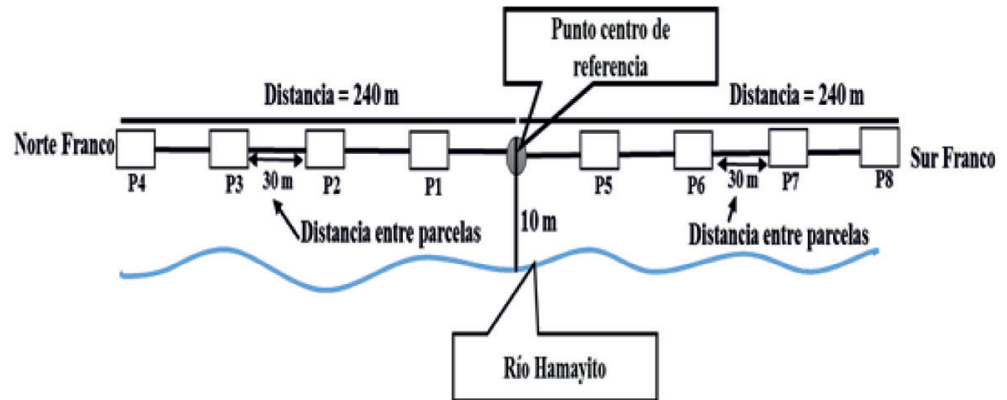

gura 2. Diseño de muestreo utilizado en el bosque de galería de la finca

\section{Variables dasométricas evaluadas}

Diámetro normal. Según Serrano y Toledo (2003), esta variable se mide a una altura de $1.30 \mathrm{~m}$ sobre el nivel del suelo en los individuos mayores o iguales a $10 \mathrm{~cm}$ y se aplicó en la parcela de $20 \mathrm{~m}$ x $30 \mathrm{~m}$. Se midió con cinta diamétrica en centímetros. Esta variable se toma para calcular el área basal del árbol.

Área basal. Instrumento útil para calcular el potencial de un bosque para recuperarse. Es una aproximación del área de la sección transversal de un árbol a la altura del diámetro normal. Se deduce de la ecuación del círculo, y se calcula por la siguiente formula (Prodan et al., 1997): 
$\mathrm{AB}=0.7854 * \mathrm{DN}^{2}$

Donde: $\mathrm{AB}=$ Área basal expresado en $\mathrm{m}^{2}$ y $\mathrm{DN}=$ Diámetro del árbol a $1.3 \mathrm{~m}$ del suelo, medido en centímetros.

Altura total. La altura total de los árboles fue determinada por medio de la observación. Para ello se tomó como guía la altura de algunos árboles medidos con el Clinómetro (SUUNTO) a una distancia de $15 \mathrm{~m}$ del árbol. Se realizaron dos lecturas la primera en la base del árbol y la segunda en el ápice terminal de la copa del árbol. Posteriormente se utilizó la siguiente fórmula matemática (Prodan et al, 1997).

$$
H=\left(\frac{\mathrm{L} 1 \pm \mathrm{L} 2}{100}\right) * \mathrm{DH}
$$

Donde: L1= Lectura 1, en la base del árbol, expresada en porcentaje, L2= Lectura 2, en la copa del árbol, expresada en porcentaje y $\mathrm{DH}=$ Distancia horizontal en metros.

Identificación de especies. Se contó con el apoyo de un baqueano el cual proporcionó el nombre común de las especies. Se colectó una muestra de cada especie y se alcoholizaron para preservarlas y ser enviadas al Herbario de la Universidad Nacional Agraria en donde se procedió a revisarla para saber su nombre científico.

\section{Variables silviculturales}

Presencia de lianas. Son plantas trepadoras delgadas y alargadas propias de las selvas tropicales. Se evaluó en los árboles mayores o iguales a $10 \mathrm{~cm}$ de diámetro normal, utilizando las categorías establecidas (Calero y Valerio, 1994 Citado por González y Narváez, 2005).

Categoría 1: son árboles que están limpios y no presentan lianas. Categoría 2: son árboles que están cubiertos de lianas en el fuste.

Categoría 3: son árboles que están cubiertos de lianas en la copa.

Categoría 4: son árboles que están cubiertos de lianas en el fuste y la copa.

Calidad de fuste. Se refiere al grado de calidad en la forma, salud y rectitud que presentan los fustes de los árboles (Synnott, 1991 como se cita en Perla y Tórrez, 2008) Se clasifican en las siguientes categorías:

Categoría 1. Se refiere a todos los árboles con fustes completamente rectos, sin daños visibles, ni enfermos.

Categoría 2. Son los árboles que presentan fustes con alguna curvatura leve, daños leves.

Categoría 3. Son los árboles que presentan fustes curvos, daños evidentes o podridos en la base o tronco. La calificación de la calidad del fuste se hizo con base a las observaciones y a los criterios establecidos por Synnott (1991), como se cita en Perla y Tórrez (2008).

Índice de valor de importancia (IVI). Es usado para comparar diferentes comunidades en base a las especies que obtienen los valores más altos y que se consideran son las de mayor importancia ecológica dentro de una comunidad en particular. Este índice resulta de la suma de los valores relativos de la abundancia, la frecuencia y la dominancia (Lamprecht, 1990).

\section{RESULTADOS Y DISCUSIÓN}

Composición florística. Con relación a la vegetación fustal se registró un total de 55 especies agrupadas en 29 familias y 51 géneros, en un área total muestreada de 1.92 ha. Según el número de árboles encontrados sobresalen las especies: Guazuma ulmifolia, y Gliricidia sepium en el bosque secundario, Thouinidium decandrum y Guazuma ulmifolia en el bosque de galería, Azadirachta indica, Albizia niopoides y Haematoxylum brasiletto en el sistema silvopastoril.

La vegetación latizal en los diferentes usos del suelo está formada por 21 familias, 33 géneros, 35 especies y 532 individuos. Las especies que sobresalen son: en el bosque secundario Stemmadenia obovata y Lonchocarpus minimiflorus, en el bosque de galería, Melicoccus bijugatus, Calycophyllum candidissimum y en el sistema silvopastoril Azadirachta indica, Stemmadenia obovata. Las familias que sobresalen según el número de especies fueron: en el bosque secundario Fabaceae y Boraginaceae (cuatro especies cada una), en el bosque de galería Fabaceae y Boraginaceae (cuatro especies cada una) y en el sistema silvopastoril la Fabaceae (cuatro especies).

La vegetación brinzal en los usos del suelo está formada por 14 familias, 21 géneros, 23 especies y 152 individuos. Las especies que sobresalen son: Lonchocarpus minimiflorus y Senna atomaria en el bosque secundario; Stemmadenia obovata y Maclura tinctoria en el bosque de galería, y Stemmadenia obovata y Azadirachta indica en el sistema silvopastoril. Las familias que sobresalen según el número de especies fueron: Fabaceae (4 especies) y Boraginaceae (tres especies) en el bosque secundario, Fabaceae (cuatro especies) y Boraginaceae (3 especies) en el bosque de galería y Meliaceae (dos especies) en el sistema silvopastoril.

\section{Estructura de la vegetación arbórea en los tres usos del suelo}

Distribución diamétrica de la vegetación fustal. En la Figura 3, se observa la distribución del número de árboles por clase diamétrica de los árboles mayores a $10 \mathrm{~cm}$ de diámetro 
normal (fustales) y se refleja un mayor número de individuos en las categorías menores y menor número de individuos en las categorías mayores.

En este sentido el mayor número de individuos presentes en los usos del suelo de la finca agroecológica Tonantzín, se encuentran en la clase diamétrica 10-19.9 cm con 201.04 árboles ha $^{-1}(76.89 \%)$ en el bosque secundario, 164.58 árboles $\mathrm{ha}^{-1}(47.30 \%)$ en el bosque de galería y con 81.25 árboles ha ${ }^{-1}(51.32 \%)$ en el sistema silvopastoril. Los individuos con diámetros mayores se agrupan en las restantes categorías diamétricas sumando el $23.11 \%$ en bosque secundario, $52.7 \%$ en bosque de galería y el $48.68 \%$ en el sistema silvopastoril.

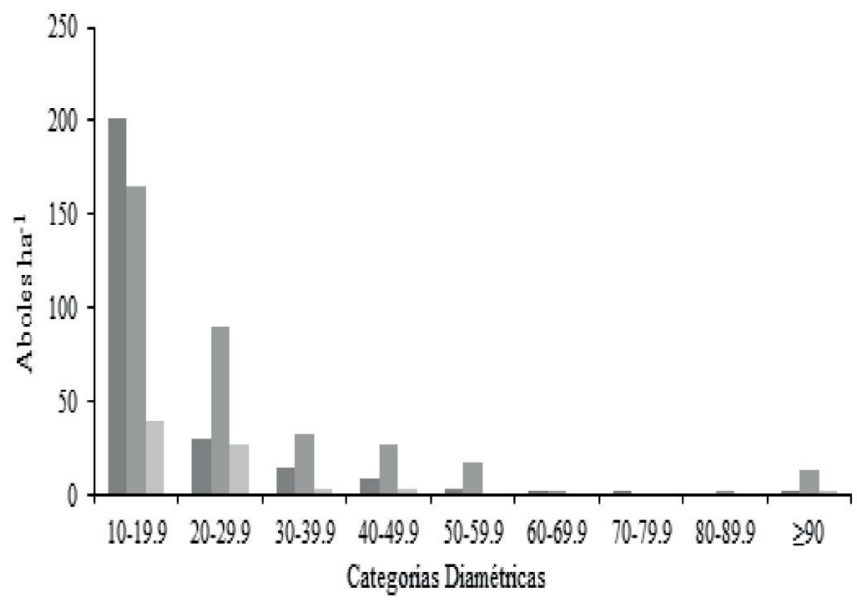

-Bosque Secundario $\backsim$ Bosque de galeria $\backsim$ Sistema Silvopastoril

Figura 3. Distribución del número de árboles por hectárea por clases diamétrica en tres usos del suelo de la finca agroecológica Tonantzín, Diriamba-Carazo, 2016.

Distribución diamétrica del área basal. En la Figura 4, se muestra la información respecto al área basal por categoría diamétrica de la vegetación presente en los usos del suelo, lo que indica que muestra una estructura casi regular en las 9 categorías diamétricas, exceptuando la última categoría en el bosque de galería, el cual presenta un valor alto de área basal por hectárea en relación a las demás categorías debido a que en el bosque de galería se encontraron árboles con diámetros superiores (Ceiba pentandra, Albizia saman, Albizia niopoides y Ficus maxima) por lo tanto mayor área basal.

\section{Parámetro de la estructura vertical}

Distribución por clase de altura. La Figura 5 muestra la distribución del número de árboles por hectárea por clases de altura, el cual indica que el bosque secundario presenta el mayor número de árboles por hectárea en las clases de 5-9.9 m y 10-14.9 m, con el 94.42 \% (246.88 árboles ha-1). Para el bosque de galería en las clases 5-9.9 m y 10-14.9 m, con el $83.83 \%$ (291.66 árboles ha $\left.{ }^{-1}\right)$.
En lo que refiere al sistema silvopastoril el mayor número de árboles por hectárea se encontró en la categoría 5-9.9m, con el $60 \%$ (93.75 árboles ha $\mathrm{ha}^{-1}$ ). Las demás categorías representan el $5.58 \%$ (14.58 árboles ha $\left.{ }^{-1}\right)$ en el bosque secundario, el $16.16 \%$ (56.25 árboles ha-1) en el bosque de galería y el $39.98 \%$ (62.47 árboles ha-1) en el sistema silvopastoril.

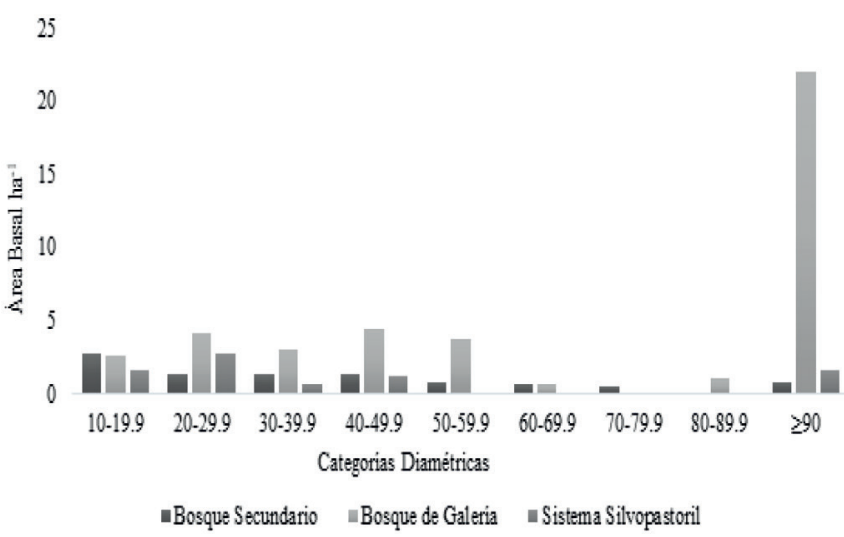

Figura 4. Distribución del área basal por hectárea por clases diamétricas en tres usos del suelo de la Finca Agroecológica Tonantzín Diriamba-Carazo, 2016.

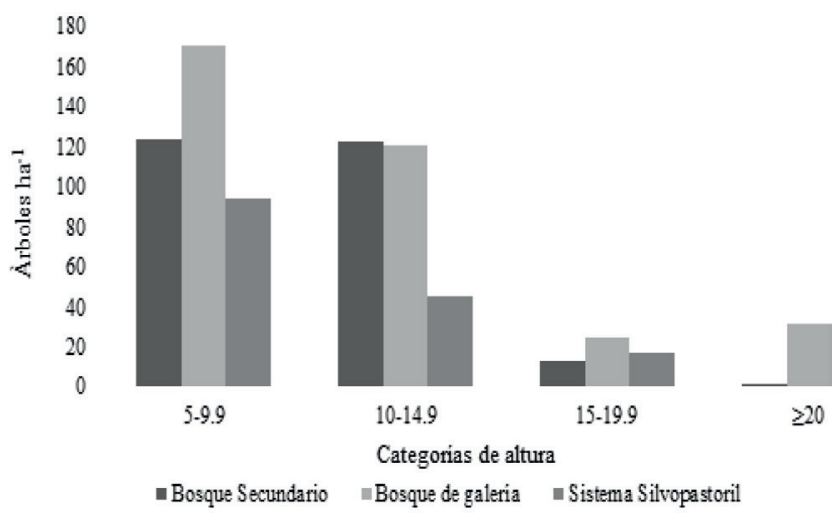

Figura 5. Distribución del número de árboles por hectárea por clase de altura en tres usos del suelo de la Finca Agroecológica Tonantzín, Diriamba-Carazo, 2016.

Estado silvicultural de la vegetación con $D N \geq 10 \mathrm{~cm}$, en los tres usos del suelo Calidad de fuste. En el bosque secundario, se encontró que 144.79 árboles ha-1 $(55.38 \%)$ presentaron fuste con curvatura y daño leve, 47.92 árboles ha $^{-1}(18.33 \%)$ presentaron fuste enfermo, quebrado $\mathrm{y}$ podridos en la base y el tronco y 68.75 árboles ha ${ }^{-1}$ (26.29 $\%)$ presentan fuste recto sin ningún daño ni enfermos. Sin embargo, como se trata de un área con fines de reserva, los árboles mal formados tienen que ser dejados para que cumplan con su ciclo bilógico y los árboles muy enfermos (muertos) se pueden utilizar como leña para uso de la casa. 
En el bosque de galería, se encontró que 156.25 árboles ha-1 $(44.91 \%)$ presentaron fuste con curvatura y daño leve, el 143.74 árboles ha ${ }^{-1}$ (41.32\%) presentan fuste recto sin ningún daño ni enfermos y 47.92 árboles ha $^{-1}(13.77 \%)$ presentaron fuste enfermo, quebrado y podridos en la base y el tronco. En este caso como se trata del bosque de galería, se pueden mantener los árboles ya que sirven de protección a la fuente de agua. En lo relativo al sistema silvopastoril se encontró que 95.83 árboles ha-1 (61.33\%) de los individuos muestreados presentan fuste recto sin ningún daño ni enfermos, 60.42 árboles ha ${ }^{-1}(38.67 \%)$ presentaron fuste con curvatura y daño leve. Por otro lado, no se encontró ningún individuo ubicado en la categoría 3. En este caso los árboles están sano y levemente curvos, por lo tanto, estos pueden ser utilizados como fuentes semilleras para incidir en mejoramiento de la regeneración natural.

Presencia de lianas. El bosque secundario se encontró que un $66.53 \%$ (173.96árboles $\mathrm{ha}^{-1}$ ) de los individuos se encuentran libre de lianas, el $15.14 \%$ presentan $(39.58$ árboles ha ${ }^{-1}$ ) lianas en el fuste, el $11.95 \%$ (31.25 árboles $\left.\mathrm{ha}^{-1}\right)$ presentan lianas en la copa y solamente el $6.37 \%$ (16.67 árboles $\mathrm{ha}^{-1}$ ) de los individuos presentan lianas en el fuste y la copa. Existen un $33.46 \%$

Cuadro 2. Índice de valor de importancia (IVI) de las cinco especies más importantes en el bosque secundario de la finca agroecológica Tonantzín, Diriamba, Carazo, 2016

\begin{tabular}{lccccccc}
\hline Especie & \multicolumn{2}{c}{ Abundancia } & \multicolumn{2}{c}{ Frecuencia } & \multicolumn{2}{c}{ Dominancia } & IVI \\
\hline Bosque secundario & Absoluta & $\%$ & Absoluta & $\%$ & Absoluta & $\%$ & $\%$ \\
Guazuma ulmifolia & 46.87 & 17.95 & 0.72 & 11.77 & 3.0131 & 31.91 & 20.56 \\
Gliricidia sepium & 42.70 & 16.33 & 0.24 & 3.92 & 0.5972 & 6.33 & 8.85 \\
Mimosa arenosa & 26.04 & 9.96 & 0.30 & 4.90 & 0.3975 & 4.21 & 6.35 \\
Homalium racemosum & 16.66 & 6.37 & 0.42 & 6.86 & 0.4201 & 4.45 & 5.89 \\
Stemmadenia obovata & 16.66 & 6.37 & 0.48 & 7.84 & 0.1974 & 2.09 & 5.43 \\
\hline Sub total & 148.96 & 56.95 & 2.16 & 35.29 & 4.6253 & 48.98 & 47.06 \\
\hline Otras sp (32 sp) & 112.50 & 43.02 & 3.96 & 64.71 & 4.8204 & 51.03 & 52.92 \\
\hline Total 47 sp & 261.44 & 100 & 6.12 & 100 & 9.4457 & 100 & 10 \\
\hline
\end{tabular}

(87.5 árboles ha $\left.^{-1}\right)$ de árboles afectados por lianas, lo que indica que el bosque está siendo poco afectado por la presencia de lianas, por lo tanto, el bosque no requiere de intervenciones silviculturales, si se considera que no hay muchos árboles afectados por la presencia de lianas (C. Calero, comunicación personal, 14 de marzo 2017).

Con respecto al bosque de galería, se encontró que el $79.64 \%$ (277.08 árboles ha $\left.{ }^{-1}\right)$ de los árboles se encuentran libre de lianas, el $14.97 \%$ (52.08 árboles ha $\left.{ }^{-1}\right)$ presentan lianas en el fuste, el $4.19 \%$ (14.58 árboles ha' $\left.{ }^{-1}\right)$ presentan lianas en la copa y solamente el $1.20 \%$ (4.16 árboles ha $\left.{ }^{-1}\right)$ de los individuos presentan lianas en el fuste y la copa. En este sentido existe un $20.36 \%$ de árboles afectados por la presencia de lianas.

Índice de valor de importancia (IVI) de las especies arbóreas en los tres usos del suelo de la finca Agroecológica Tonantzín. En el Cuadro 2, se muestran en orden las cinco especies con mayor índice de valor de importancia para el bosque secundario. En este sentido, Guazuma ulmifolia ocupa el primer lugar en el índice de valor de importancia por tener el valor más alto de dominancia relativa, seguido por Gliricidia sepium y Mimosa arenosa por su alta abundancia relativa, así mimo, Homalium racemosum por tener altos valores en los tres parámetros y Stemmadenia obovata el cual ocupa el último lugar dentro de las primeras cinco especies por sus valores de abundancia y frecuencia relativa.
Los valores del índice de valor de importancia generados en este trabajo son cercanos al valor reportado por Narváez (2012), el cual expresa que las especies ecológicamente más importante son Gliricidia sepium (Madero negro) y Myrospermum frutescens (Chiquirin) con 17.3 y 10.4 respectivamente, pero no son las más abundantes quedando determinado su valor de importancia por la dominancia relativa que poseen estas especies ya que la frecuencia relativa de las 10 principales especies tiene el mismo valor. Los valores del índice de importancia para las especies Gliricidia sepium, Myrospermum frutescens, Casearia corymbosa, Lonchocarpus minimiflorus y Stemmadenia obovata señalan que están bien representadas en el bosque.
En cuanto al bosque de galería, en el Cuadro 3 se muestran las cinco especies con mayor índice de valor de importancia. En este sentido Ceiba pentandra tiene el primer lugar en el índice de valor de importancia por tener el valor más alto de dominancia relativa, seguido por Cupania guatemalensis por tener altos valores en los tres parámetros, Ficus maxima por la dominancia relativa, Guazuma ulmifolia y Thouinidium decandrum por tener altos valores en los tres parámetros.

Considerando a González y Narváez (2005), hacen mención que en el bosque de galería en la hacienda Las Mercedes en el departamento de Managua, las especies arbóreas con mayor importancia ecológica son: Guazuma ulmifolia (28.22\%) y Albizia saman (19.14\%) y en Los Sábalos, Ceiba pentandra (30.67\%).

En lo relativo al sistema silvopastoril, en el Cuadro 4 se muestran las cinco especies con mayor índice de valor de importancia. En este sentido Azadirachta indica presenta el índice de valor de importancia más alto por presentar altos valores en los tres parámetros, seguido por Enterolobium cyclocarpum por la dominancia relativa, Guazuma ulmifolia, Haematoxylum brasiletto y Albizia niopoides por la abundancia y frecuencia relativa que estas presentan.

Benítez (2016) desarrollo un estudio llamado composición florística de árboles de la finca agroecológica de Zamorano, Valle El Yeguare, Honduras, destacando 
las especies más importantes del ecosistema se encontró Guazuma ulmifolia $48.07 \%$ muy utilizada en potreros de Centroamérica, seguido de Quercus oleoides $33.98 \%$ y Luehea candida con $30.94 \%$. Esto se debe a que estas especies tienen gran abundancia de individuos y dominancia en el ecosistema. La especie con el IVI más bajo dentro de las diez más importantes es Spondias purpurea con $7.96 \%$.

\section{CONCLUSIONES}

La caracterización de la vegetación arbórea registró en los tres usos del suelo un total de 29 familias, 51 géneros y 55 especies para fustales, predominando las familias Fabaceae, Sapindaceae y Malvaceae, mostrando mayor número de especies el bosque de galería y el bosque secundario por las condiciones ecológicas favorables tales como la humedad relativa en el suelo y la disponibilidad de semillas por la presencia de árboles productores de semillas.

Cuadro 3. Indice de valor de importancia (IVI) de las cinco especies más
bosque de galería de la finca agroecológica Tonantzín, Diriamba Carazo, 2016

\begin{tabular}{|c|c|c|c|c|c|c|c|}
\hline Especie & \multicolumn{2}{|c|}{ Abundancia } & \multicolumn{2}{|c|}{ Frecuencia } & \multicolumn{2}{|c|}{ Dominancia } & IVI \\
\hline Bosque de galería & Absoluta & $\%$ & Absoluta & $\%$ & Absoluta & $\%$ & $\%$ \\
\hline Ceiba pentandra & 4.17 & 1.20 & 0.12 & 2.18 & 7.2253 & 17.36 & 6.93 \\
\hline Cupania guatemalensis & 27.08 & 7.79 & 0.30 & 5.43 & 2.2035 & 5.29 & 6.17 \\
\hline Ficus maxima & 4.16 & 1.19 & 0.06 & 1.08 & 6.4141 & 15.41 & 5.89 \\
\hline Guazuma ulmifolia & 20.83 & 5.99 & 0.3 & 5.44 & 2.4141 & 5.80 & 5.74 \\
\hline $\begin{array}{l}\text { Thouinidium } \\
\text { decandrum }\end{array}$ & 22.92 & 6.59 & 0.24 & 4.35 & 1.5264 & 3.66 & 4.86 \\
\hline Sub total & 79.16 & 22.76 & 1.02 & 18.48 & 19.7834 & 47.52 & 29.59 \\
\hline Otras sp (38 sp) & 268.75 & 77.24 & 4.5 & 81.52 & 21.8492 & 52.48 & 70.41 \\
\hline Total $43 \mathrm{sp}$ & 347.91 & 100.00 & 5.52 & 100.00 & 41.6326 & 100.00 & 100.00 \\
\hline
\end{tabular}

Cuadro 4. Índice de valor de importancia (IVI) de las cinco especies más importantes en el sistema silvopastoril de la finca agroecológica Tonantzín, Diriamba-Carazo, 2017

\begin{tabular}{lccccccc}
\hline \multicolumn{1}{c}{ Especie } & \multicolumn{2}{c}{ Abundancia } & \multicolumn{2}{c}{ Frecuencia } & \multicolumn{2}{c}{ Dominancia } & IVI \\
\hline \multicolumn{1}{c}{ Sistema Silvopastoril } & Absoluta & $\%$ & Absoluta & $\%$ & Absoluta & $\%$ & $\%$ \\
Azadirachta indica & 83.33 & 53.34 & 0.42 & 33.34 & 4.0734 & 51.96 & 49.20 \\
Enterolobium & 4.16 & 2.67 & 0.12 & 9.53 & 1.8600 & 23.73 & 11.97 \\
cyclocarpum & & & & & & & \\
Guazuma ulmifolia & 14.58 & 9.34 & 0.18 & 14.29 & 0.3675 & 4.69 & 9.43 \\
Haematoxylum brasiletto & 16.66 & 10.66 & 0.12 & 9.52 & 0.2818 & 3.59 & 7.92 \\
Albizia niopoides & 16.66 & 10.66 & 0.06 & 4.76 & 0.5173 & 6.59 & 7.34 \\
\hline Sub-total & 135.39 & 86.64 & 0.90 & 71.44 & 7.1000 & 90.53 & 82.86 \\
\hline Otras sp (6 sp) & 20.83 & 13.33 & 0.36 & 28.57 & 0.3551 & 9.44 & 17.11 \\
\hline Total 47 sp & 156.22 & 100.00 & 1.26 & 100.00 & 7.4551 & 100.00 & 100.00 \\
\hline
\end{tabular}

Los valores del índice de importancia para las especies Guazuma ulmifolia, Gliricidia sepium para el bosque secundario, así como Ceiba pentandra, Cupania guatemalensis en el bosque de galería y Azadirachta indica, Enterolobium cyclocarpum para el sisma silvopastoril se encuentran en el ecosistema con una buena representación. Esto favorece a estas especies, y por lo tanto no tendría algún tipo de amenazada natural, cualquier cambio en la variabilidad climática o por la aplicación de un tratamiento silvicultural.

Cabe señalar que en los usos del suelo existen especies arbóreas con gran importancia ecológica que con un adecuado manejo garantizaran la permanencia de las características del ecosistema forestal. Así mismo el comportamiento de la diversidad biológica dentro de cada uso de suelo va a depender de la biología y ecología de estas especies (Armas y Meneces, 2007).
El sistema silvopastoril registró menor número de especies, debido algunos factores limitantes como presencia de ganado, pastoreo, incidencia de incendios, extracción de leña y de postes por las comunidades aledañas.

En los tres usos del suelo en la categoría latizal se registró un total de 42 familias, 63 géneros y 69 especies. Mientras que en la categoría brinzal se registró un total 24 familias, 33 géneros y 36 especies. Las familias más predominantes en los tres usos de suelo fueron Fabaceae y Boraginaceae.

La mayoría de los individuos se encuentran en buenas condiciones de calidad de fuste, dominados por las categorías 1 y 2 . Así mismo, la mayor parte de los individuos se encuentran libre de lianas en los tres usos del suelo.

La vegetación arbórea en los tres usos del suelo se encuentra de manera general en un buen estado de regeneración natural según su distribución diamétrica en forma de " $\mathrm{j}$ invertida".

Las especies arbóreas con mayor importancia ecológica (IVI) presentes en los usos del suelo son: Guazuma ulmifolia en el bosque secundario, Ceiba pentandra en el bosque de galería y Enterolobium cyclocarpum en el sistema silvopastoril.

\section{AGRADECIMIENTOS}

Al señor Señor Humberto Solórzano propietario de la Reserva Agroecológica Tonanzint por recibirnos en la finca, compartiendo su espacio, tiempo y conocimientos. A la Dirección de Investigación, Extensión y Postgrado de la Universidad Nacional Agraria quien nos dio la oportunidad de aplicar y quedar seleccionados en los fondos para proyecto en la convocatoria 2016. A la administración y autoridades de la Facultad de Recursos Naturales y del Ambiente por su incondicional apoyo durante la ejecución de la investigación. 


\section{REFERENCIAS BIBLIOGRÁFICAS}

Aguilar, D. C. y Ramos, S. A. (2016). Caracterización de fauna silvestre con fines ecoturísticos de la finca agroecológica Tonantzín en Diriamba, Carazo [Tesis de pregrado, Universidad Nacional Agraria]. Repositorio Institucional UNA. https://repositorio.una.edu. ni/3862/1/tnp01a283.pdf

Alvis, J.F. (2009). Análisis estructural de un bosque natural localizado en zona rural del municipio de Popayán. Biotecnología en el sector agropecuario y agroindustrial, 7(1), 115-122. http://www.scielo.org.co/pdf/bsaa/v7n1/v7n1a13.pdf

Armas, H. E. y Meneces, E. A. (2007). Composición, Diversidad, Estructura e importancia de las Especies Arbóreas y Palmas del Bosque Seco de la Finca "Rosita", Reserva Natural Estero Padre Ramos, Chinandega, Nicaragua [Tesis de pregrado, Universidad Nacional Agraria]. Repositorio Institucional UNA. https://repositorio.una.edu.ni/1108/1/tnk10a727.pdf

Benítez Nassar, D. (2016). Composición florística de árboles de la finca agroecológica de Zamorano, Valle El Yeguare, Honduras [Tesis de pregrado, Universidad Zamorano]. Repositorio Institucional UNA. https://bdigital.zamorano.edu/bitstream/11036/5720/1/IAD2016-T007.pdf

González, H. y Narváez, S. (2005). Diagnóstico del bosque de galería de la Hacienda Las Mercedes, Managua [Tesis de pregrado, Universidad Nacional Agraria]. Repositorio Institucional UNA. https://repositorio.una.edu.ni/1071/1/tnk10g643d.pdf

Instituto Nacional Forestal. (2009). Resultados del Inventario Nacional Forestal: Nicaragua, 2007-2008. https://rb.gy/g9xlpf

Lamprecht, H. (1990). Silvicultura en los Trópicos. Eschborn; GTZ.

Narváez Espinoza, O. (2012). Dinámica de crecimiento, estructura y composición de la vegetación secundaria en Trópico Seco de Nandarola, Nicaragua [Tesis de maestría, Universidad Nacional Agraria]. Repositorio Institucional UNA. https://repositorio.una. edu.ni/1183/1/tnk10n238.pdf

Ministerio de Recursos Naturales y del Ambiente. (2001). Informe del estado ambiental en Nicaragua, 2001. IMPRIMATUR. https:// rb.gy/wdz22w

Organización de las Naciones Unidas para la Alimentación y la Agricultura. (2004). Actualización de la evaluación de los recursos forestales mundiales a 2005: Términos y Definiciones. http://www.fao.org/3/a-ae156s.pdf

Perla, C. y Tórrez, J. (2008). Caracterización de la vegetación forestal, usos y diversidad de especies de la vegetación forestal en la reserva privada Escameca Grande, San Juan del Sur, Rivas [Tesis de pregrado, Universidad Nacional Agraria]. Repositorio Institucional UNA. https://repositorio.una.edu.ni/1115/1/tnp01p451.pdf

Pezo, D. y Ibrahim, M. (1999). Sistemas silvopastoriles (2da ed.). CATIE. https://rb.gy/zzsjhb

Prodan, M., Peters, R., Cox, F. y Real, P (1997). Mensura Forestal. GTZ; IICA. http://repiica.iica.int/docs/B4179e/B4179e.pdf

Sáenz, G. y Finegan, B. (2000). Monitoreo de la regeneración natural con fines de manejo forestal. CATIE

Serrano, J. y Toledo, C. (2003). Estado estructural y silvicultural de la especie endémica Ocotea strigosa van der Werff (Arrayán), circundante a la laguna Miraflor, Miraflor, Esteli, 2002 [Tesis de pregrado, Universidad Nacional Agraria]. Repositorio Institucional UNA. https://repositorio.una.edu.ni/1026/1/tnk10s487.pdf

Scott, Ch. (1991). Optimal design of a plot cluster for monitoring. USDA. https://www.ncrs.fs.fed.us/pubs/jrnl/1993/ne_1993_ scott-c_001.pdf 